ENGELbERT KöTteR

\section{Das große GU PraxisHandbuch Kräuter}

Gräfe und Unzer Verlag GmbH, München, 2009, 288 Seiten, zahlreiche farbige Abbildungen, gebunden, ISBN 978-3-8338-1129-6; 24,90€

Wo pflanze ich mein Basilikum? Welche Arten harmonieren mit meinem Rosmarin, und was bekommt ihm gar nicht? Ist das Süßblatt wirklich süß, und schmeckt Lakritz-Tagetes nach Lakritz? Und was ist eigentlich Ulluco?

Diese und andere Fragen werden im „Großen GU PraxisHandbuch Kräuter" beantwortet. Es enthält Informationen rund um Aussaat, Pflege, Ernte, Konservierung und Verwendung von über 400 Kräuterarten.

Im ersten Teil des Buches wird ausführlich auf alle Besonderheiten bei der Anzucht der Kräuter eingegangen. Dabei wird die Auswahl der Böden, des richtigen Düngers und der Pflanzgefäße abgehandelt. Dem Kräuterliebhaber wird erklärt, welches die angemessene Wassermenge und der optimale Schnitt ist und es werden Tipps für die Überwinterung genannt. Als besonders nützlich erweisen sich zwei Doppelseiten, die sich den gängigsten Kräuterschädlingen und -krankheiten widmen. Diese und die entsprechenden Gegenmittel werden aufgezeigt.

Das nächste Kapitel beschäftigt sich ausführlich mit der Verwendung der Kräuter. Ein Augenmerk liegt dabei auf Möglichkeiten ihres Verzehrs, wobei in verschiedenen Rezepten Tees, Kaltgetränke und andere Leckerbissen vorgestellt werden - auch die Frankfurter Grüne Soße ist dabei. Darüber hinaus findet man Anregungen zur Herstellung von Duftbouquets, kosmetischen Produkten und Kräuterbädern.

Der umfassendste Teil des Buches besteht aus Pflanzenporträts. Diese enthalten neben einer aussagekräftigen Abbildung zahlreiche
Hinweise zur Pflege der Kräuter, Tipps zur Ernte und zur Verwendung. Durch eine eingängige Symbolik (z. B. volle Gießkanne = viel gießen, Sonne $=$ bevorzugt einen sonnigen Standort) erhält der Leser bereits auf den ersten Blick die wichtigsten Grundinformationen. Auch die Hinweise, welche Pflanzenteile geerntet und wie sie verwendet werden können, fallen dem Leser direkt ins Auge. Wer genauer nachliest, erhält aus einer detaillierten Beschreibung der Arten die nötigen Informationen, um die Pflanzen optimal zu pflegen. Die Rubrik „Wissenswertes" liefert die eine oder andere Anekdote zur Namensherkunft oder erklärt den volksmedizinischen Nutzen der einzelnen Arten. Neben den gängigen und bekannten Kräutern werden auch viele Exoten vorgestellt, die langsam den Einzug in die heimische Küche antreten. Ein abschließendes Glossar erklärt die wichtigsten Termini rund um Anbau und Biologie der Kräuter.

Das „Große GU PraxisHandbuch Kräuter" ist ein empfehlenswertes Nachschlagewerk für alle Hobbygärtner und Köche, die ihr Essen gerne mit frischen Kräutern verfeinern.

Eileen Wasner 